\title{
Efeito do envelhecimento no controle executivo na tarefa de geração aleatória de números
}

\author{
Amer Cavalheiro Hamdan \\ Universidade Federal do Paraná
}

\begin{abstract}
RESUMO
A diminuição de habilidades relacionadas ao Controle Executivo - CE em idosos tem sido relacionada com o envelhecimento neurobiológico do Sistema Pré-Frontal. Este estudo comparou a pontuação obtida no teste de Geração Aleatória de Números (RNG) por dois grupos: 30 jovens universitários acima de 18 anos e 36 idosos acima de 60 anos. Este último grupo foi dividido de acordo com o critério escolaridade em até 8 anos ou mais de 8 anos. Os resultados mostram que os jovens obtiveram média de 0,344 $\pm 0,04$ no índice do RNG; os idosos com até 8 anos de escolaridade pontuaram 0,432 $\pm 0,10$ e os idosos com 9 anos ou mais alcançaram média $0,393 \pm 0,05$. A análise estatística destes dados sugere que o desempenho de idosos no teste do RNG $(0,420 \pm 0,09)$ foi significativamente pior quando comparado ao desempenho dos jovens ( $\mathrm{t}(64)=-3,9927 ; \mathrm{p}=0,0001)$, confirmando que as habilidades de manipulação e de controle inibitório da informação relacionada ao CE estão diminuídas nos idosos, provavelmente devido ao processo natural de envelhecimento do Córtex Frontal.
\end{abstract}

Palavras-chave: neuropsicologia; envelhecimento; funções executivas

\begin{abstract}
Aging effect on Executive Control in the Random Number Generation Test

The decline of abilities related to Executive Control -EC with aging has been related with the PreFrontal System neurobiological aging. This study compared the score obtained in the Random Number Generation Test (RNG) by two groups: 30 college students (older than 18) and 36 elderly (older than 60). This last group was divided according to the criteria of schooling in up to 8 years of schooling and over 8 years of schooling. The results show that the younger participants scored $0,344 \pm$ 0,04 in the RNG test; the elderly with up to 8 years of schooling punctuated $0,432 \pm 0,10$ and that those who studied for 9 years or more reached a mean of $0,393 \pm 0,05$. The statistical analysis of these data suggest that the elderly performance in the RNG test $(0,420 \pm 0,09)$ was significantly worse when compared to the performance of the younger participants $(t(64)=-3,9927 ; p=0,0001)$, which confirms that the manipulation and inhibitory control abilities of information are decreased in older people, probably due to the natural aging process of the Frontal Cortex.
\end{abstract}

Keywords: neuropsychology; aging; executive functions

O Controle Executivo - CE é um mecanismo regulador cujo produto envolve as habilidades de planejamento, iniciação, seguimento e automonitoramento de comportamentos dirigidos a um fim; comportamentos esses genericamente chamados de Funções Executivas. Distúrbios neurocomportamentais do CE podem ocorrem por lesões nas regiões pré-frontais ou mesmo nas conexões entre regiões pré-frontais e outras estruturas corticais ou subcorticais (Benson \& Miller, 1997; Estévez-Gonzáles, Garcia-Sánches \& Barraquer-Boras, 2000; Royall e cols, 2002; Tekin \& Cummings, 2002).
Teoricamente, o CE pode ser explicado por três posições: a) como componente da memória operacional (Baddeley, 1996), b) como resultado de processos inibitórios (Dempster, 1992) e c) como resultado de ambos os processos (Diamond \& Taylor, 1996). Neste trabalho assume-se a concepção de que o CE tem a função básica de manutenção on-line (função retrospectiva) e de manipulação (função prospectiva) da informação. Essas funções são mediadas por processos inibitórios que regulam a interferência de novas e antigas informações. Deste modo, as habilidades tradicionalmente relacionadas às funções executivas, tais como, atenção, abstração, planejamento, flexibilidade 
mental, autocontrole e memória operacional (Loring, 1999; Spreen \& Strauss, 1998), seriam gerenciadas por mecanismos de manutenção e manipulação reguladas por processos inibitórios da informação.

No processo natural de envelhecimento do ser humano ocorre uma diminuição geral das habilidades cognitivas e, especialmente, das habilidades relacionadas ao CE, tais como: atenção dividida, organização e seguimento de ações e controle e manipulação de informações (Bryan \& Luszcz, 2000; Fisk \& Warr, 1996; Grady \& Craik, 2000; Green, 2000; Mejia, Pineda, Alvarez \& Ardila, 1998; Nielson, Langenecker \& Garavan, 2002; Parkin, 1999; Troyer, Moscovitch \& Winor, 1997; Wecker, Kramer, Wisniewski, Delis \& Kaplan, 2000; Woodruff-Pak, 1997; Zelazo, Craik \& Booth, 2004). Uma possível explicação para essas alterações seria o processo de envelhecimento neurobiológico do Sistema Pré-Frontal e do sistema dopaminérgico (Daselaar, Veltman, Rombouts, Raaijmakers \& Jonker, 2003; MacPherson, Phillips \& Della Sala, 2002; Morcom, Good, Frackowiak \& Rugg, 2003; Stebbins e cols, 2002; West, 1996). Contudo, na literatura poucos estudos têm investigado empiricamente as mudanças relacionadas ao $\mathrm{CE}$ ao longo do processo de envelhecimento (Kray, Eber \& Lindenberger, 2004). No Brasil, também existem poucos estudos (Avila \& Miotto, 2003; Hamdan \& Bueno, 2005; Saboya, Franco \& Mattos, 2002).

Alguns estudos têm sugerido que o Teste de Geração Aleatória de Números - RNG é um instrumento eficiente na avaliação do CE (Hamdan \& Bueno, 2005; Hamdan, Souza \& Bueno, 2004; Jahanshahi \& Dirnberg, 1999; Jahanshahi, Dirneberg, Fuller \& Frith, 2000; Jahanshahi e cols, 1998). O objetivo desta pesquisa foi realizar uma investigação empírica para comparar o desempenho de jovens e idosos no Teste de Geração Aleatória de Números - RNG para avaliar o efeito do envelhecimento no controle executivo. A hipótese inicialmente investigada foi de que os idosos teriam um desempenho pior do que os jovens no teste do RNG.

\section{MÉTODO}

\section{Participantes}

Participaram deste estudo 30 jovens universitários, acima de 18 anos, de ambos os sexos, recrutados entre estudantes do curso de Psicologia da Universidade Federal de Mato Grosso do Sul - UFMS, e 36 idosos, acima de 60 anos, de ambos os sexos, selecionados a partir de uma amostra de 91 idosos submetidos à ava- liação neuropsicológica. Para diminuir a influência da escolaridade na comparação entre o grupo de idosos e grupo de jovens, o grupo de idosos foi dividido em dois sub-grupos: 25 idosos pertencentes ao grupo com $\leq 08$ anos de escolaridade e 11 idosos pertencentes ao grupo com $>08$ anos de escolaridade. Todos os idosos freqüentavam as atividades físicas e sociais promovidas pelo Centro de Convivência de Idosos em Campo Grande, MS.

Os critérios de exclusão para o grupo de idosos foram: 1) idade abaixo de 60 anos; 2) presença de déficit visual ou auditivo não corrigido; 3) histórico de alcoolismo ou exposição a toxinas; 4) uso crônico de medicamentos psicoativos (benzodiazepínicos, neurolépticos etc.); 5) presença de anormalidades endócrinas e metabólicas; 6) alterações neurológicas (parkinsonismo, traumatismo crâneo-encefálico, doença cerebrovascular etc.); 7) distúrbios psiquiátricos (distúrbios de ansiedade, distúrbios de pensamento etc.) e 8) indivíduos analfabetos. Os critérios de exclusão foram garantidos através de uma avaliação obtida mediante entrevista semi-estruturada conduzida pelo próprio autor.

\section{Procedimentos e instrumentos de coletas de dados}

Os idosos foram selecionados tendo como critério o desempenho no Mini Exame do Estado Mental MEEM (Folstein, Folstein \& Mchugh, 1975) e na Escala Clínica de Demência - CDR (Morris, 1993). Para os idosos com até 08 anos de escolaridade utilizamos o ponto de corte para o MEEM de $\geq 24$ pontos e para os idosos com mais de 08 anos de escolaridade foi utilizado o ponto de corte $\geq 26$ tendo como referência os estudos realizados no Brasil (Almeida, 1998; Brucki, Nitirni, Carmelli, Bertolucci \& Okamoto, 2003). Os idosos de ambos os grupo tiveram pontuação 0 no CDR (sem demência). Todos os participantes assinaram o Termo de Consentimento de Participação, previamente aprovado pelo Comitê de Ética da Universidade Federal de São Paulo (UNIFESP).

A tarefa de Geração Aleatória de Números (Random Number Generation - RNG) consiste em solicitar aos participantes para falar números aleatórios de 1 a 10 , numa sequiência de 100 números. A velocidade de resposta para geração de números foi de um número por segundo, controlada por meio de um metrônomo. A pontuação foi realizada através do Índice de Aleatoriedade Subjetiva do RNG, proposto por Evans (1978). Este índice mede através de um logaritmo o grau de distribuição de números aleatórios gerados - 
quanto mais próximo o índice de 1 menor o grau de aleatoriedade dos números. Para realizar a tarefa do RNG, foram lidas e discutidas com os participantes as instruções, conforme apresentado em trabalho anterior (Hamdan, Souza \& Bueno, 2004).

\section{Critérios de análise dos dados}

Os resultados obtidos foram comparados entre as amostras de jovens e idosos. Para a análise descritiva dos resultados utilizamos as medidas de tendência central (média e mediana) e de dispersão (desviopadrão). De acordo, com dados na literatura, assumese que as propriedades da distribuição dos resultados satisfazem os critérios de normalidade (Cepeda, Kramer \& Gonzáles de Sather, 2001; Kray, Eber \& Lindenberger, 2004; Zelazo, Craik \& Booth, 2004). Para a análise inferencial empregamos medidas de estimativa (intervalo de confiança) e provas paramétricas (Teste t, Análise de Variância - ANOVA, Teste de Correlação de Pearson) para comparar o desempenho e verificar a associação dos resultados. $\mathrm{O}$ valor $\mathrm{p}$ foi estabelecido para cada teste e a hipótese nula foi rejeitada no nível de significância de $\alpha=5 \%$.

\section{RESULTADOS}

A idade dos trinta estudantes $(n=30)$ universitários apresentou média de 23,9 $\pm 5,2$ anos e IC $(95 \%)$ de 21,9 e 25,8 anos. A média do grupo de idosos com até 8 anos de escolaridade $(n=25)$ foi de $70,1 \pm 5,2$ anos e IC (95\%) de 67,9 e 72,2 anos. O grupo de idosos, com mais de 08 anos de escolaridade $(n=11)$, apresentou média de $66 \pm 7$ anos e IC (95\%) de 61,2 e 70,7 anos. Os três grupos diferiram significativamente quanto à idade, ANOVA, $\mathrm{F}(2,65)=536,07 ; \mathrm{p}<0,05$. A Tabela 1 apresenta as características demográficas da amostra.

\begin{tabular}{lccccc}
\multicolumn{6}{l}{ Tabela 1. Características demográficas da amostra } \\
\begin{tabular}{lccccc}
\multicolumn{7}{c}{ Jovens } & Idosos1 & Idosos2 & $F$ & $\mathrm{p}$ \\
& $M \pm D P$ & $M \pm D P$ & $M \pm D P$ & & \\
\hline $\mathrm{n}(\mathrm{f} / \mathrm{m})$ & $30(13 / 27)$ & $25(23 / 02)$ & $11(09 / 02)$ & & \\
Idade $^{*}$ & $23,9 \pm 5,2$ & $70,1 \pm 5,2$ & $66 \pm 07$ & 536,07 & 0,000 \\
\hline
\end{tabular}
\end{tabular}

Nota. $n=$ número de participantes; $f=$ feminino; $m=$ masculino; ${ }^{\text {significativo. }}$

A média do Índice do RNG de jovens foi de $0,344 \pm 0,04$ com IC $(95 \%)$ de 0,327 e 0,360 . Para o grupo de idosos $\leq 08$ anos de escolaridade, a média do Indice do RNG foi de $0,432 \pm 0,10$ e IC $(95 \%)$ de 0,387 e 0,477 . Os idosos com $\geq 9$ anos obtiveram média no Índice do RNG de $0,393 \pm 0,05$ e IC $(95 \%)$ de 0,355 e 0,428 . A comparação das diferenças das médias entre jovens e todos os idosos no desempenho do RNG foi estatisticamente significante, $\mathrm{t}(64)=-3,9927 ; \mathrm{p}=$ 0,0001 . As diferenças entre as médias dos grupos em função da escolaridade também foi estatisticamente significante, $F(2,65)=9,08 ; p=0,000$. O Teste de Tukey revelou diferenças apenas entre jovens e idosos com até 08 anos de escolaridade, mas não com idosos com mais de 08 anos de escolaridade (Figura 1). O Teste de Correlação de Pearson revelou associação significativa entre idade X Índice do RNG; resultando valores de $r=0,448$ e $p=0,000$ e também entre o Índice do RNG de idosos com $\leq 8$ anos $X$ com $\geq 9$ anos de escolaridade; em que $r=0,605$ e $p=0,004$.

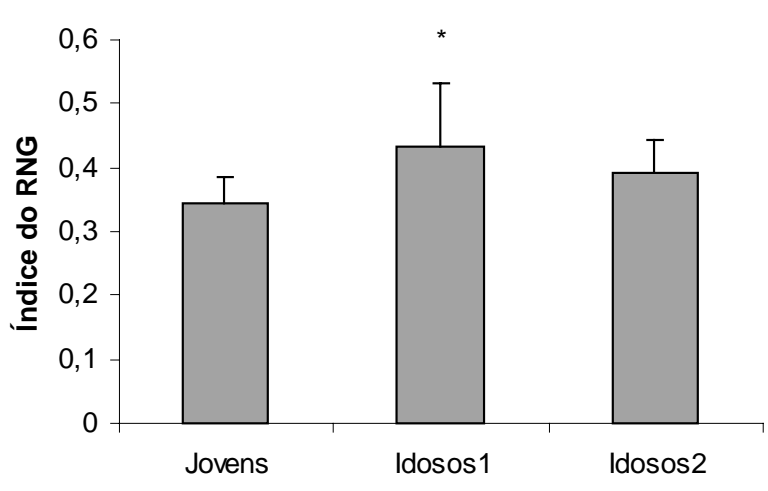

Figura 1. Desempenho de jovens e idosos na Tarefa de Geração Aleatória de Números. 


\section{DISCUSSÃO}

O presente estudo foi realizado com o objetivo de avaliar o controle executivo através da comparação do desempenho de jovens e idosos no Teste de Geração Aleatória de Números - RNG. Neste experimento observou-se que o desempenho de idosos na tarefa do RNG foi significativamente pior quando comparado ao desempenho dos jovens, confirmando a hipótese inicialmente levantada de que o CE declina com o processo de evelhecimento. Estes dados são consistentes com as evidencias encontradas na literatura de que as habilidades de manipulação e de controle inibitório da informação diminuem no processo natural do envelhecimento (Hamdan \& Bueno, 2005; Nielson, Langenecker \& Garavan, 2002; Van Der Linden, Beerten \& Pesent, 1998).

Estas diferenças estão relacionadas à diminuição da capacidade de Controle Executivo, dos processos inibitórios e de manipulação de números, nos idosos. Provavelmente, estas dificuldades nos idosos ocorrem pelo processo de envelhecimento do Córtex Frontal (Stebbins \& cols, 2002; West, 1996; Zelazo, Craik \& Booth, 2004). Estudos de neuroimagem sugerem que a produção de números aleatórios no teste do RNG exige considerável demanda do $\mathrm{CE}$, ativando as regiões do Córtex Pré-Frontal Dorsolateral (Daniels, Witt, Wolff, Jansen \& Deuschl, 2003; Jahanshahi \& Dirnberg, 1999; Jahanshahi, Dirnberg, Fuller \& Frith, 2000; Jahanshahi \& cols., 1998).

Em resumo, a presente investigação confirmou a visão de que o CE declina com o processo de envelhecimento. Por outro lado, o efeito do envelhecimento não é um processo linear, como se evidenciou pela diminuição deste efeito pelo maior grau de escolaridade dos idosos. As diferenças entre jovens e idosos no RNG ocorrem devido a dificuldades maiores dos idosos em inibir respostas estereotipadas na geração de números, processos estes que são modulados pelo Córtex Pré-Frontal Dorsolateral. Contudo, a variável escolaridade pode diminuir estas diferenças.

Uma limitação da abrangência deste estudo referese a questão metodológica. Estudos transversais são potencialmente suscetíveis de diferenças de coorte, através de variáveis não controladas, que interferem nas variáveis investigadas. Em decorrência deste fato, estudos longitudinais são necessários para corroborar os resultados encontrados.

\section{REFERÊNCIAS}

Almeida, O. P. (1998). Mini-exame do Estado Mental e o diagnóstico de demência no Brasil. Arquivos de Neuropsiquiatria, 56 (3-B), 605-612.

Avila, R \& Miotto, E. (2003). Funções executivas no envelhecimento normal e na doença de Alzheimer. Jornal Brasileiro de Psiquiatria, 52 (1), 53-63.

Baddeley, A (1996). Exploring the central executive. The Quarterly Journal of Experimental Psychology. 49A, 5-28.

Benson, D. F. \& Miller, B. L. (1997). Frontal lobes: clinical and anatomic aspects. Em Freinberg, T. D. \& Farah, M. J. (Org.), Behavioral Neurology and Neuropsychology. (pp. 401-418). New York: McGraw-Hill.

Bryan, J. \& Luszcz, M. A. (2000). Measurement of executive function: considerations for detecting adult age differences. Journal of Clinical and Experimental Neuropsychology, 22 (1), 40-55.

Brucki, S. M. D.; Nitrini, R.; Caramelli, P.; Bertolucci, P. H. F. \& Okamoto, I. H. (2003). Sugestões para o uso do Mini-Exame do Estado Mental no Brasil. Arquivos de Neuropsiquiatria, 61 (3-B), 777-781.

Cepeda, N. J., Kramer, A. F. \& Salther, J. C. M. G. (2001). Changes in executive control across the life span: Examination of task-switchimg performance. Developmental Psychology, 37 (5), 715-730.

Daniels, C., Witt, K., Wolff, S., Jansen, O. \& Deuschl, G. (2003). Rate dependency of the human cortical network subserving executive functions during generation of random number series - A functional magnetic resonance imaging study. Neuroscience Letters, 345, 25-28.

Daselaar, S. M., Veltman, D. J., Rombouts, S. A. R. B., Raaijmakers, J. G. W. \& Jonker, C. (2003). Neuroanatomical correlates of episodic encoding and retrieval in young and elderly subjects. Brain, 126, 43-56.

Dempster, F. N. (1992). The rise and fall of the inhibitory mechanism: toward a unified theory of cognitive development and aging. Developmental Review, 12, 45-75.

Diamond, A. \& Taylor, C. (1996). Developmental of an aspect of executive control: development of the abilities to remember what I said and to "Do as I say, not as I do". Developmental Psychbiology, 29, 315-334.

Estévez-Gonzáles, A., Garcia-Sánchez, C. \& Barraquer-Boras, L. I. (2000). Los lóbulos frontales: El cérebro ejecutivo. Revista de Neurología, 31 (6), 566-577.

Evans, F. J. (1978). Monitoring attention deployment by random number generation: an index to measure subjective randomness. Bulletin of the Psychonomic Society, 12 (12), 35-38.

Fisk, J. E. \& Warr, P. (1996). Age and working memory: the role of perceptual, the central executive, and the phonological loop. Psychology and Aging, 11 (2), 316-323.

Folstein, M. F., Folstein, S. E. \& Mchugh, P. R. (1975). Minimental state: a practical method for grading the cognitive state of patients for the clinician. Journal of Psychiatric Research, 12 (3), 189-198.

Grady, C. L. \& Craik, F. I. M. (2000). Changes in memory processing with age. Current Opinion in Neurobiology, 10, 224-231.

Interação em Psicologia, Curitiba, jul./dez. 2006, (10)2, p. 267-271 
Green, J. (2000). Neuropsychological evaluation of the older adult: a clinician's guidebook. San Diego: Academic Press.

Hamdan, A. C., Souza, J. A. \& Bueno, O. F. A. (2004). Performance of university students on Random Number Generation at different rates to evaluate executive functions. Arquivos de Neuropsiquiatria, 62 (1), 58-60.

Hamdan, A. C. \& Bueno, O. F. A. (2005). Relações entre controle executivo e a memória episódica no comprometimento cognitivo leve e na demência tipo Alzheimer. Estudos de Psicologia, 10 (1), 63-71.

Jahanshahi, M. \& Dirnberg, G. (1999). The left dorsolateral prefrontal cortex and random number generation of responses: studies with transcranial magnetic stimulation. Neuropsychologia, 37, 181-190.

Jahanshahi, M., Dirnberg, G., Fuller, R. \& Frith, C. D. (2000). The role of the dorsolateral prefrontal cortex in random number generation: a study with Positron Emission Tomography. Neuroimage, 12 (6), 713-724.

Jahanshahi, M., Profice, P., Brown, R. G., Ridding, M. C., Dirnberg, G. \& Rothwell, J. C. (1998). The effects of transcranial magnetic stimulation over the dorsolateral prefrontal cortex on suppression of habitual counting during random number generation. Brain, 121, 1533-1544.

Kray, J., Eber, J. \& Lindenberger, U. (2004). Age differences in executive functioning across the lifespan: the role of verbalization in task preparation. Acta Psychologica, 115, 143-165.

Loring, D. (1999). INS Dictionary of Neuropsychology. Oxford University Press: New York.

MacPherson, S. E., Phillips, L. H. \& Della Sala, S. (2002). Age, executive function, and social decision making: a dorsolateral prefrontal theory of cognitive aging. Psychology and Aging, 17 (4), 598-609.

Mejia, S., Pineda, D., Alvarez, L. M. \& Ardila, A. (1998). Individual differences in memory and executive function abilities during normal aging. International Journal of Neuroscience, 95, 271-284.

Morcom, A. M., Good, C. D., Frackowiak, R. S. J. \& Rugg, M. D. (2003). Age effects on the neural correlates of successful memory encoding. Brain, 126, 213-229.

Morris, J. C. (1993). The Clinical Dementia Rating (CDR) current version and scoring rules. Neurology, 43, 2412-2414.

Nielson, K. A., Langenecker, S. A. \& Garavan, H. (2002). Differences in the functional neuroanatomy of inhibitory control across the adult life span. Psychology and Aging, 17 (1), 56-71.
Parkin, A. J. (1999). Exploraciones en neuropsicología cognitiva. Madrid: Editorial Medica Panamericana.

Royall, D. R., Lauterbach, E. C., Cummings, J. L., Reeve, A., Rummans, T. A., Kaufer, D. I., LaFrance, W.C. \& Coffey, C. E. (2002). Executive Control Function: a review of its promise and challenges for clinical research. A report from the Committee on Research of the American Neuropsychiatric Association. Journal of Neuropsychiatry Clinical Neuroscience, 14 (4), 377-405.

Saboya, E., Franco, C. A. \& Mattos, P. (2002). Relações entre processos cognitivos nas funções executivas. Jornal Brasileiro de Psiquiatria, 52 (2), 91-100.

Spreen, O. \& Strauss, E. (1998). A compendium of neuropsychological tests. Administration, norms, and commentary. New York: Oxford University Press.

Stebbins, G. T., Carrillo, M., Dorfman, J., Dirksen, C., Desmond, J. E., Turner, D. A., Bennett, D. A., Wilson, R. S., Glover, G. \& Gabrieli, J. D. E. (2002). Aging effects on memory encoding in the frontal lobes. Psychology and Aging. 17 (1), 44-55.

Tekin, S. \& Cummings, J. L. (2002). Frontal-subcortical neuronal circuits and clinical neuropsychiatry: an update. Journal of Psychosomatic Research, 53, 647-654.

Troyer, A. K., Moscovitch, M. \& Winor, G. (1997). Clustering and switching as two components of verbal fluency: Evidence from younger and older healthy adults. Neuropsychology, 11 (1), 138-146.

Van der Linden, M., Beerten, A. \& Pesenti, M. (1998). Agerelated differences in Random Generation. Brain and Cognition, 38 (1), 1-16.

Wecker, N. S., Kramer, J. H., Wisniewski, A., Delis, D. C. \& Kaplan, E. (2000). Age effects on executive ability. Neuropsychology, 14 (3), 409-414.

West, R. L. (1996). An application of prefrontal cortex function Theory to Cognitive Aging. Psychological Bulletin, 120 (2), 272-292.

Woodruff-Pak, D. (1997). The neuropsychology of aging. Oxford: Blackwell.

Zelazo, D. P., Craik, F. I. M \& Booth, L. (2004). Executive function across the life span. Acta Psychologica, 115, 167-183.

Recebido: 30/03/2006 Revisado: 16/07/2006 Aceito: 02/11/2006

\section{Sobre o autor:}

Amer Cavalheiro Hamdan: Doutor em Psicobiologia. Professor Adjunto no Departamento de Psicologia da Universidade Federal do Paraná - UFPR

Endereço para correspondência: achamdan@ufpr.br. 